\title{
An Anomaly Detection Algorithm Based on Frequency Change of Satellite Telemetry Data
}

\author{
Qin Bo, Yang Yumin, Wang An, Gong Xizhong \\ Key Laboratory of Spacecraft in-orbit Fault Diagnosis and Maintenance Xi'an Satellite Control Center Xi'an, China \\ Cooler2003@sina.com
}

\begin{abstract}
Based on different data frequency's characteristics, design the algorithms of distinguishing cycle data's margin apart and distinguishing cycle data's monotone apart, to monitor satellite's downlink data and early warning abnormal. Compared with rapid fourier transform, this algorithm was proved to be reasonable and efficient.
\end{abstract}

Keywords—telemetry data; frequency; parallel computing

\section{INTRODUCTION}

Telemetry data frequency is one of the important symbols of satellite state. Whether most of satellite telemetry output are normal or not is correlated with the frequency. For example, the analog quantity output of the CTU and RTU for data management subsystem of a satellite is 4 second of cycle in normal situation. And it has disordered cycle and irregular wave in failure situation. The output current of solar wing and bearing temperature normally are change stabilized. When it is abnormal, it will change severely. At present, aiming at the detection of frequency change for satellite telemetry data, the domestic and foreign studies mainly focus on comparing the historical data statistics and model constructing forecast to identify the characteristic change, such as adopting and so on. According to the practical engineering application, a detection algorithm based on periodical data deference detection and data monotony detection is proposed, which can balance the complication of the algorithm and detection effect an abnormal situation, and improve the response speed and ability an abnormal situation..

\section{FREQUENCY FEATURE CLASSIFICATION}

The satellite is usually divided into public system and private system. The utility system generally refers to the application independent part, such as the structure of the system, thermal control system, power supply system, the attitude and orbit control system, wireless measure and control system, data management system etc.. The special system is a satellite payload. Payload resources satellite is all kinds of sensors, including visible light cameras, multispectral camera, multi spectral scanners, and synthetic aperture radar.

The meteorological satellite payload includes scanning radiometer, infrared spectrometer, vertical atmospheric detector and atmospheric temperature detector [4]

Telemetry system is a part of satellite control system. Usually the working condition of satellite telemetry data, the frequency characteristic main performance for the following situations:
1) frequency constant. As the frequency changes, then the telemetry parameter characterizing the physical component is abnormal. The telemetry parameters of typical, such as working state of computer switching circuit for attitude and orbit control, normal for 4 second cycle square wave.

2) The frequency of non-constant. Namely frequency constant is not equal to a value, such as equal to the value that a component is abnormal. Such as signal onboard computer seconds must appear in the high and low level, the gyro output angular velocity must also be changing is the normal state. As the frequency of 0 , part a problem.

3) The frequency changes some complex constraints. Do not meet the constraints, also represent abnormal. As the satellite radiometer radio-meter CHRPT output and DPT output, there must be change in continuous 5 frames, and the amplitude of variation range is 0 to $5 \mathrm{~V}$; otherwise, explain the telemetry data anomaly.

\section{DESIGN OF DETECTION ALGORITHM}

Design of frequency change detection algorithm, is a comprehensive engineering. On the one hand, to the extent possible, ensure the detection accuracy, which can not only avoid the omission, but also to reduce the false alarm. On the other hand, consider the real-time requirement, the algorithm execution costs as low as possible, and to eliminate the influence of sampling frequency and the frequency of the data it conflicts

\section{A. The sampling process analysis}

Spectrum of sampled signal, is the original analog signal spectrum to sample angular frequency of periodic, periodic ext ension and. That is to say, the original analog signal spectrum along the frequency axis, each interval sampling frequency rep eated once, on the formation of the spectrum of sampled signal [5] as shown in Figure 1

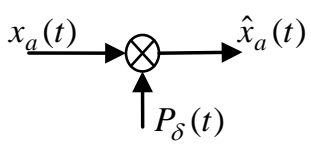

Figure. 1 Schematic diagram of the sampling process In Figure $1, x_{a}(t)$ as the input analog signal, $P_{\delta}(t)$ as the unit impulse string, $\hat{x}_{a}(t)$ as sample output. Design

$$
P_{\delta}(t)=\sum_{n=-\infty}^{\infty} \delta(t-n T)
$$




$$
\hat{x}_{a}(t)=x_{a}(t) \bullet P_{\delta}(t)=\sum_{n=-\infty}^{\infty} x_{a}(t) \delta(t-n T)
$$

In the impulse signal units (when $t=n T$, then $\delta(t$ ) may have a non-zero value) case, the relationship between available sampling signal and the input signal, the output signal (3) design:

$$
\hat{x}_{a}(t)=\sum_{n=-\infty}^{\infty} x_{a}(n T) \delta(t-n T)
$$

Design

$$
\begin{aligned}
X_{a}(j \Omega) & =F T\left[x_{a}(t)\right] \\
\hat{X}_{a}(j \Omega) & =F T\left[\hat{x}_{a}(t)\right] \\
P_{\delta}(j \Omega) & =F T\left[P_{\delta}(t)\right]
\end{aligned}
$$

Type FT shows in the Fourier transform of the signal. Ta ke

$$
\Omega_{s}=2 \pi / T, a_{k}=\frac{1}{T} \int_{-T / 2}^{T / 2} \delta(t) e^{-j k \Omega_{s} t} d t=\frac{1}{T}
$$

By formula (1) know, type (6) can be converted into

$$
P_{\delta}(j \Omega)=\sum_{k=-\infty}^{\infty} 2 \pi a_{k} \delta(\Omega-k \Omega)
$$

That is

$$
\begin{aligned}
\hat{X}_{a}(j \Omega) & =\frac{1}{2 \pi} X_{a}(j \Omega) \cdot P_{\delta}(j \Omega) \\
& =\frac{1}{2 \pi} \cdot \frac{2 \pi}{T} \int_{-\infty}^{\infty} X_{a}(j \theta) \sum_{k=-\infty}^{\infty} \delta\left(\Omega-k \Omega_{s}-\theta\right) d \theta \\
& =\frac{1}{T} \sum_{k=-\infty}^{\infty} \int_{-\infty}^{\infty} X_{a}(j \theta) \delta\left(\Omega-k \Omega_{s}-\theta\right) d \theta \\
& =\frac{1}{T} \sum_{k=-\infty}^{\infty} X_{a}\left(j \Omega-j k \Omega_{s}\right)
\end{aligned}
$$

Therefore, in order to set values in the cycle, the least common multiple period sampling output signal can be found by sampling cycle and cycle of data that.

A cycle $T_{0}$ ( $T_{0}=6$ seconds) of the sawtooth wave, through periodic $T_{1}$ ( $T_{1}=5$ seconds)sampling, forming a cycle $T_{2}$ ( $T_{2}=30$ seconds) of the discrete wave. As shown in figure 2 。

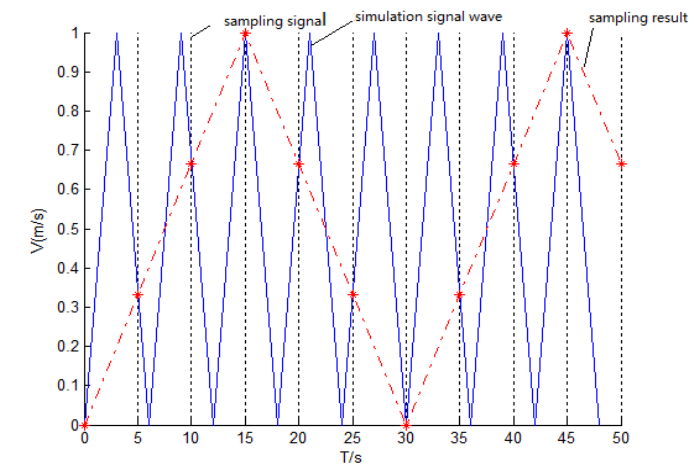

Figure 2 sampling, analog signals and the results of sampling cycle

\section{B. Temporal characteristics analysis}

In order to facilitate the analysis, the discussion is divided into the following several kinds of situations:

1) Frequency and amplitude are given the value of the telemetry data

According to Section 2.1 for a discussion of sampling, simulation and results of sampling wave cycle relationship, known frequency and amplitude are fixed value and the telemetry data is known, in the same sampling period change cycle and data, data and any of its the data value of the difference was 0 ; in the sampling period and the change cycle consistent, taking the least common multiple and, then the data and any of its office data value difference is 0 , as shown in figure 3.

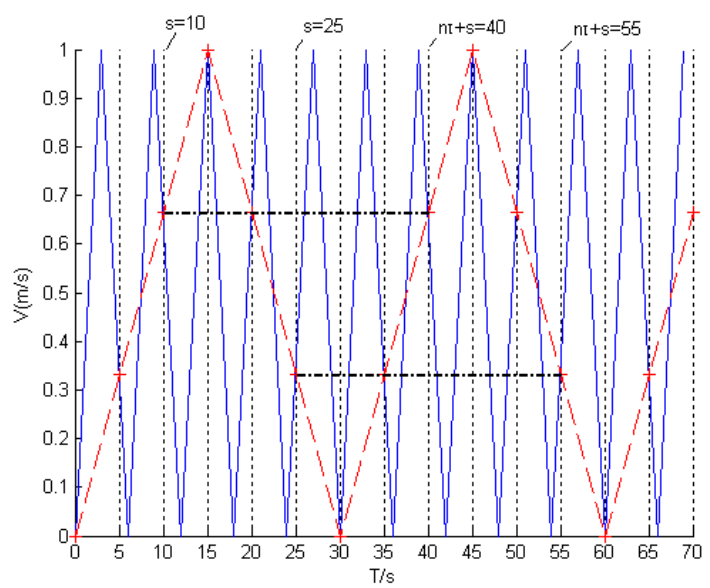

Figure 3 frequencies for the timing characteristics of known constant

In engineering application, because of various kinds of interference errors, can judge whether a difference less than minimum $\zeta$ (usually desirable amplitude $1 / 10$ ), but are not limited to, is equal to 0 .

2) Frequency and fixed amplitude change of telemetry data

The known frequency is constant and amplitude change of circumstances, the telemetry data sampling cycle and cycle change consistency in the data, by the characteristic of periodic waveform, arbitrary and known data numerical monotonic consistency; when the sampling period and the change cycle is inconsistent, taking the least common multiple and, then the data and the data of arbitrary numerical monotonicity is consistent, with monotonic rise or fall, the tangent slope absolute value increases with the increase of data waveform amplitude. As shown in figure 4. 


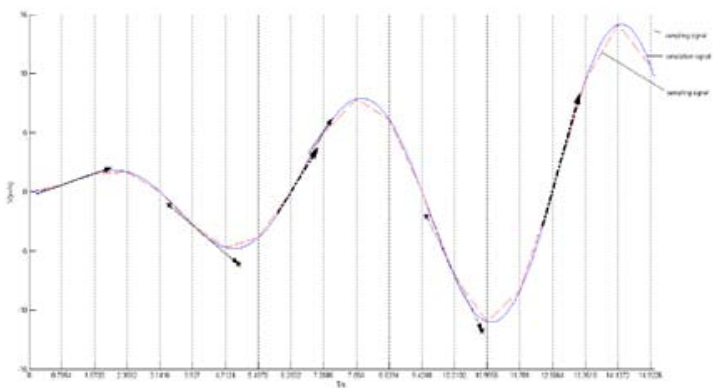

Figure 4 frequency and fixed amplitude is not fixed when the timing characteristics

\section{Design of detection algorithm}

Combined with the 2.1 section, 2.2 section analyses, we can draw the conclusion: in the frequency under the condition of invariable, arbitrary interval $n \tau(n, \tau)$ of analog signal and the sampling signal cycle of the least common multiple of two telemetry data, the difference is 0 , or monotonic consistency. Therefore, the following frequency changes detection algorithm design of telemetry data:

1) telemetry data preprocessing the received, continuous $\phi(\phi \geq 3)$ data points were calculated for each data point with the previous data points of the time interval, the time interval for average value, $\boldsymbol{\varepsilon}$ approximate value as the data sampling period;

2) The least common multiple of data $\boldsymbol{\varepsilon}_{\text {sampling period }}$ $T$ and the known cycle of change $\tau$;

3) From the beginning of the current discrete data points, in order to take back $([\tau]+1)(+1$ rounding) discrete point, into array $\mathrm{B}$;

4) For each data point in the array $B$, for it with the backward distance $\tau$ data value difference;

5) If the timing convergence all difference, the telemetry parameters in the $2 \tau$ cycle period of time frequency is not changed, otherwise turn 6);

5) Arbitrary array in $\mathrm{B}$ points $[\alpha, \beta]$, calculation corresponding to each point backward data point $[\mu, v]$ distance $\tau$, if $\alpha-\beta$ and $\mu-v$ sign consistent with, the telemetry parameters in the $2 \tau$ cycle period of time frequency is not changed.

From the above calculation process can be seen, the data sampling cycle calculation $\mathcal{E}$ and the difference discrimination process, are of certain amount of calculation based on the statistics. Therefore, the frequency of occurrence within short time jump, follow-up time and restore the original frequency, this algorithm adaptability.

\section{Algorithm complexity analyses}

By the 2.3 day, we can see that, in the algorithm for circular mainly focus on $\varepsilon_{\text {and }} \mathrm{B}$ data, comparison.
Upon request $\varepsilon, \phi$ data need to do $\frac{(\phi-1) \phi}{2}$ subtraction calculation; difference discrimination, a $[\tau / \varepsilon+1]$ data B, need to do $((\tau / \varepsilon+1) \tau / \varepsilon) / 2$ subtraction calculation, is the least common multiples of sampling period and known change cycle data. Therefore, the complexity of the algorithm can be approximated as $O\left(T^{2}\right)$.

\section{THE EXAMPLE ANALYSIS}

The sampling signal cycle $\varepsilon=0.5$ seconds, simulation of a satellite component voltage telemetry parameter 20 seconds of data, as shown in table 1.

Table 1 Simulation of remote parameter value

\begin{tabular}{lllllll}
\hline \multicolumn{7}{c}{ Telemetry data value $(\mathrm{V})$} \\
\hline 0 & 0.4794 & 0.8415 & 0.9975 & 0.9093 & 0.5985 \\
0.1411 & -0.3508 & -0.7568 & -0.9775 & -0.9589 & - \\
0.7055 & -0.2794 & 0.2151 & 0.6570 & 0.9380 \\
0.9894 & 0.7985 & 0.4121 & -0.0752 & -0.5440 & - \\
0.8797 & -1.0000 & -0.8755 & -0.5366 & -0.0663 \\
0.4202 & 0.8038 & 0.9906 & 0.9349 & 0.6503 \\
0.2065 & -0.2879 & 0.1499 & 0.6055 & 0.9129 & - \\
0.7118 & -0.9614 & -0.9756 & -0.7510 & -0.3425 & \\
\hline
\end{tabular}

Its essence is the sinusoidal signal frequency changes, as shown in Figure 5.

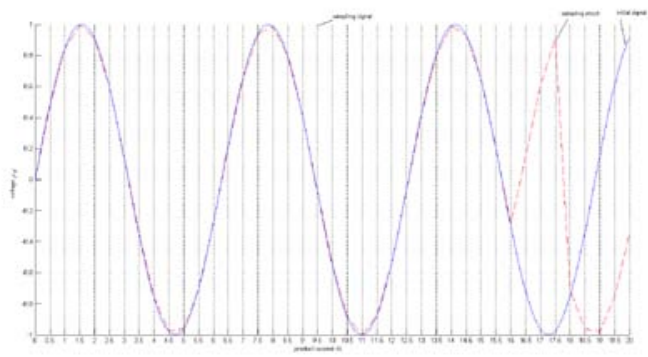

Figure 5 Simulation of remote timing sampling plan

The calculation process is as follows:

1) 3 groups of telemetry parameter, time interval were 0.5 seconds, in which the sampling period $\varepsilon=0.5$;

2) Known telemetry data cycle $T=2 \pi$, computing the least common multiple $\tau=2 \pi$, approximate value $\tau=6$;

3) Calculation of $[\tau+1]=7$;

4) According to the step 0.5 in turn $J_{s}=0,0.5,1 \ldots 13$, the following calculation:

a) From $J_{s}$ the beginning, followed by taking 7 data values into the array $\mathrm{B}$;

b) On every bit of data in B, for its backward interval $(\tau=6)$ data, stored in the array R;

c) calculates the difference of data and $\mathrm{R}$ in $\mathrm{B}$;

d) To judge the convergence. 
Through the calculation, found that when $J_{s}=7.5$, the $J_{s} \in[7.5,10.5] 7$ data values placed in array in B, namely $\mathrm{B}=\{0.9380,0.9894,0.7985,0.4121,-0.0752,-0.5440,-0.8797\}$; for every bit of data in B, for its backward interval ( $\tau=6$ ) data, stored in the array $\mathrm{R}$,namely $\mathrm{R}=\{0.8038,0.9906,0.9349,0.6503,0.2065, \quad-0.2879,0.1499\}$, and $\mathrm{R}$ data points at $\mathrm{B}$ values as shown in figure 6 .

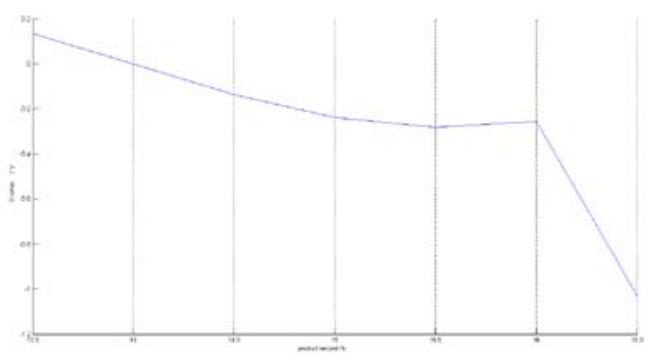

Figure. 6 schematic diagram of difference

As you can see from Figure 6, when the product for 16.5 seconds, the difference curve declined sharply, can determine this data frequency changes.

With fast Fu Liye transform, because the 41 data samples is too small, cannot detect the frequency change, as shown.

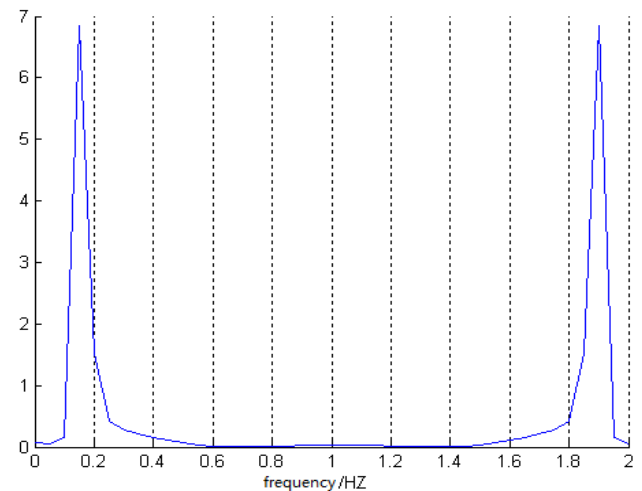

Figure. 7 Schematic diagrams of the FFT detection results

Until the number of samples is increased to 47 , the fast Fu Liye transform are shown in Figure 8.
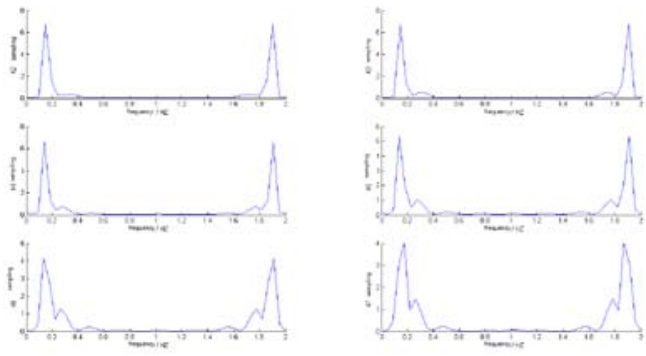

Figure. 8 schematic diagram of different samples FFT test results

Compared with the fast Fu Liye transform, this algorithm can be found earlier frequency change.

\section{CONCLUSION}

Obtain the telemetry data is one of a number of links in the process, namely has the effect of electrical characteristics, $\mathrm{A} / \mathrm{D}$ conversion, there is also the space environment, channel transmission, such as the calculation of the influence of ground. Frequency is one of the characterizations of telemetry data only less due to interference and change. Study on frequency characteristics of the telemetry data, timely early warning of abnormal analysis has important significance. This paper only discussed on change detection methods of mathematical characteristics; further research is needed to change a lot of complex frequency.

\section{ACKNOWLEDGEMENTS}

The research was partly supported by National Nature Fund of China (No.61473222).

\section{REFERENCES}

[1] Zhang Dong, Hu Shaolin, Zhang Wei et al. Satellite downlink data change detection method based on fault identification, Journal of aerospace dynamics,2011,1(1):112-115

[2] Zhang Wei, Hu Shaolin, Bruce Lee. Algorithm and application of the fault detection of seasonal index model based on the satellite downlink data. Journal of aerospace dynamics, 2011, 1(1):123-126

[3] H. Chipman, E. George, and R. McCulloch. Bayesian treed models, to appear in Machine Learning. Technical Report http://bevo2.bus.utexas.edu/GeorgeE/Research papers/treed-models.pdf, University of Waterloo, 2001.

[4] NASA Earth Satellites constitute [EB/OL]. [2012-0302]. http://www.nationalstation.org.cn/121232.html..

[5] Ding Yumei, Gao Quan. Digital signal processing [M]. Xi'an: Xi'an Electronic and Science University press,2000: 20. 